Effects of thermal annealing on the optical properties of InGaNAs/GaAs multiple quantum wells

This content has been downloaded from IOPscience. Please scroll down to see the full text. 2002 Semicond. Sci. Technol. 17145

(http://iopscience.iop.org/0268-1242/17/2/310)

View the table of contents for this issue, or go to the journal homepage for more

Download details:

IP Address: 132.174.255.116

This content was downloaded on 16/08/2015 at 03:33

Please note that terms and conditions apply. 


\title{
Effects of thermal annealing on the optical properties of InGaNAs/GaAs multiple quantum wells
}

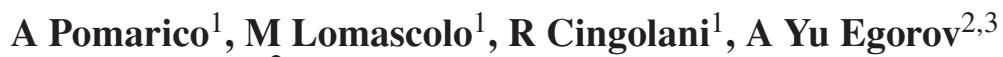 \\ and $\mathbf{H}$ Riechert $^{2}$ \\ ${ }^{1}$ National Nanotechnology Laboratory of INFM-Dipartimento di Ingegneria \\ dell'Innovazione, University of Lecce, Italy \\ ${ }^{2}$ Infineon Technologies, Corporate Research Photonics, München, Germany
}

Received 14 September 2001, in final form 12 December 2001

Published 10 January 2002

Online at stacks.iop.org/SST/17/145

\begin{abstract}
We present an optical characterization of as-grown and thermally annealed InGaNAs/GaAs multiple quantum well samples. In both samples, from the analysis of the photoluminescence spectra we can infer that the low-temperature photoluminescence emission is related to carriers localized in the alloy potential fluctuations; with increasing temperature, we observe their gradual delocalization and then the transition towards a completely different type of lineshape, typical of free carrier recombinations. The comparison between the photoluminescence spectra of the as-grown and the annealed samples shows that a remarkable improvement of the optical properties occurs after the thermal annealing. This improvement is related to an important reduction in the density of the defects and in the depth of the alloy potential fluctuations.
\end{abstract}

\section{Introduction}

In recent years, $\operatorname{In}_{x} \mathrm{Ga}_{1-x} \mathrm{~N}_{y} \mathrm{As}_{1-y}$ has emerged as a subject of intense research. By adjusting the In and $\mathrm{N}$ concentrations, InGaNAs can be grown lattice matched to GaAs, with a wide range of bandgap energies. A giant bowing coefficient for the InGaNAs bandgap leading to a redshift of luminescence with increasing $\mathrm{N}$ concentration has been demonstrated [1-4]. Owing to this peculiarity and to the large conduction band offset typical of the InGaNAs/GaAs heterostructures, this quaternary is considered as a promising material for long-wavelength lasers with high-temperature performances. Nevertheless, InGaNAs exploitation has been impeded up to now by its limited quality, which has been attributed to fluctuations in alloy composition and to $\mathrm{N}$ induced nonradiative centres [5-6]. It has been shown that this can be improved by post-growth thermal annealing of the heterostructure [7-9]. In this paper, we report on an optical characterization of as-grown and thermally annealed InGaNAs/GaAs multiple quantum well (MQW) samples. We demonstrate a number of important quality improvements

3 On leave from AF Ioffe Physico-Technical Institute, St Petersburg, Russia. occurring after thermal annealing, namely: (i) the reduction of the density of defects, (ii) the decrease of the average depth of the alloy potential fluctuations and (iii) the improvement of the homogeneity of the sample on the long range (centimetre).

\section{Experiment}

The samples investigated in this paper are InGaNAs/GaAs MQWs, with well composition and growth conditions similar to those employed in lasers with state-of-the-art performance [10]. The samples were grown by solid source molecular beam epitaxy (MBE) on (001) GaAs substrates, using an RFcoupled plasma source to generate reactive nitrogen species from $\mathrm{N}_{2}$. Growth was performed under As-stable conditions; the growth temperature of the QWs was about $430{ }^{\circ} \mathrm{C}$. The structures we examined consist of five InGaNAs QWs ( $X_{\text {In }} \approx 35 \%, X_{N} \approx 1.7 \%$ ) of $10 \mathrm{~nm}$ thickness, separated by 30 $\mathrm{nm}$ GaAs barrier layers. Below and above this MQW are $65 \mathrm{~nm}$ thick GaAs layers and this layer package is surrounded by five-period AlAs/GaAs superlattices $(2 \mathrm{~nm} / 2 \mathrm{~nm})$ for carrier confinement. The topmost AlAs layer at the same time serves to protect the structure from thermal degradation during subsequent annealing processes. 


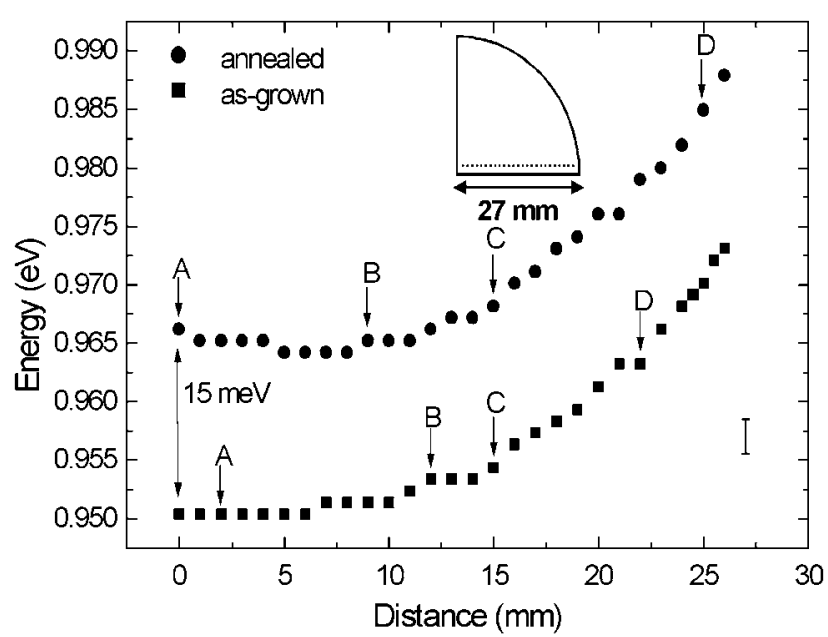

Figure 1. Peak energies of the PL spectra as a function of the position of the excitation spot along a radius of the wafer, for the as-grown and the annealed samples. The spectra were taken at room temperature. The excitation power was $15 \mathrm{~mW}$. The error bar for the experimental data is reported in the right-hand side of the figure. The arrows indicate the points investigated by temperature-dependent and excitation power-dependent spectroscopy.

Samples were cleaved in the form of stripes across the wafer, which were then cut into two symmetrical halves. One of these pieces was subsequently thermally annealed in a hydrogen atmosphere at $720{ }^{\circ} \mathrm{C}$ for $10 \mathrm{~min}$. This procedure gives similar results to in situ annealing in the growth chamber. The other piece serves as the as-grown reference sample.

The optical characterization of the as-grown sample and of the annealed sample consisted of photoluminescence (PL) measurements recorded at different positions of the wafer, under varying temperature and excitation conditions. The PL was excited by the $514 \mathrm{~nm}$ line of an argon laser, under $\mathrm{cw}$ conditions, and dispersed by a $1 \mathrm{~m}$ focal length monocromator equipped with a $\mathrm{N}_{2}$-cooled Ge detector. The excitation spot size on the samples was about $100 \mu \mathrm{m}$.

\section{Results and discussion}

The results of the room-temperature PL measurements are shown in figure 1 , where we display the PL peak energy taken every $1 \mathrm{~mm}$ along the radius of the wafer for both samples. In the annealed sample we observe a systematic blueshift of about $15 \mathrm{meV}$ with respect to the corresponding points of the as-grown sample. Such a blueshift has been reported in several studies (it may amount up to $45 \mathrm{meV}$ ) and it is attributed to a In-Ga interdiffusion at the QW interfaces $[7,8]$ or to a reconstruction at the atomic level in the InGaNAs layer [9]. For both samples the peak energy regularly increases when going towards the edge of the wafer, reaching a maximum blueshift of $22 \mathrm{meV}$ at the edge with respect to the wafer centre. This suggests a systematic decrease in the well width and/or a variation in the alloy composition (in particular, a reduction of the $\mathrm{N}$ content) when going from the centre towards the outer regions of the wafer, which results to be unaffected by the annealing.

We have carried out PL measurements as functions of temperature $T$ at four different points of the two samples

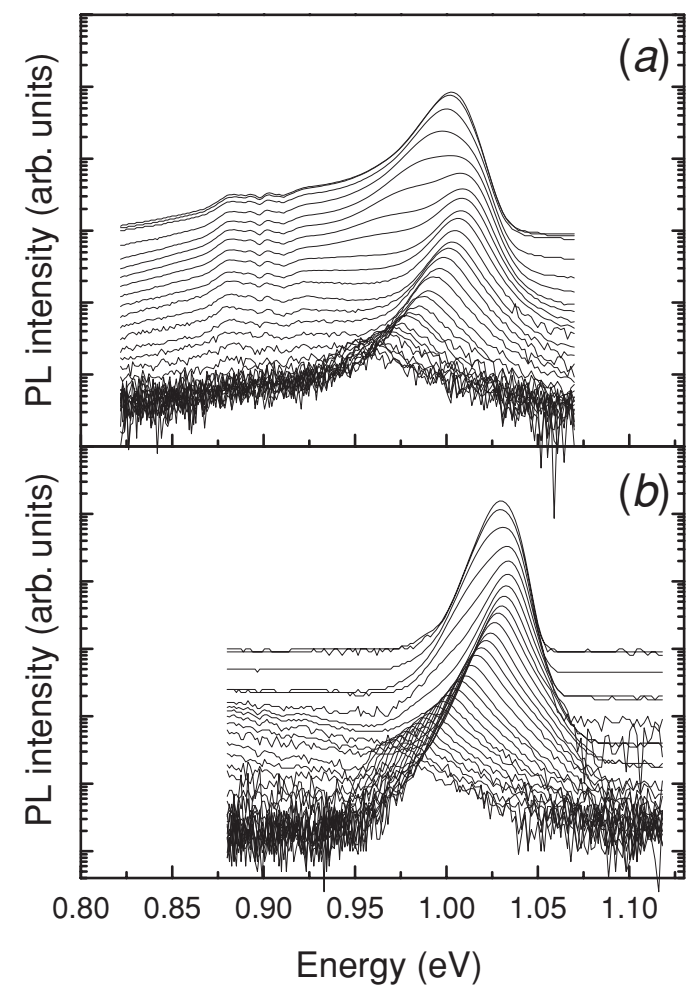

Figure 2. (a) PL spectra of the as-grown sample recorded in the point $\mathrm{A}$ at various temperatures (from $10 \mathrm{~K}$ to $300 \mathrm{~K}$ in steps of $10 \mathrm{~K}$ ). The excitation power was $42 \mathrm{~mW}$. (b) The same for point C of the annealed sample.

(positions A, B, C and D labelled in figure 1), from $10 \mathrm{~K}$ to room temperature, in steps of $10 \mathrm{~K}$. As an example, in figure 2 we show the PL spectra measured in position A of the as-grown sample and in position $\mathrm{C}$ of the annealed sample.

The $10 \mathrm{~K}$ spectrum of the as-grown sample (figure $2(a)$ ) is dominated by a broad and asymmetric emission peak, centred at $1.003-1.027 \mathrm{eV}$ (depending on the position on the wafer) and with an average full width at half maximum (FWHM) of $19 \mathrm{meV}$; the spectrum also shows a very extended lowenergy tail. With increasing $T$, the peak energy shows an initial blueshift, which ends at about $70 \mathrm{~K}$, followed by a redshift up to room temperature, following a Varshni-like law. A blueshift of the peak energy and a saturation of the tail states can be observed as well, by increasing the excitation power, at a fixed temperature (figure 3). From these data we infer that in the low-temperature conditions the main contribution to the luminescence is due to localized carriers, trapped in the potential fluctuations due to the random alloy composition; the carriers gradually delocalize with increasing $T$ and, from about $100 \mathrm{~K}$ on, the luminescence is due to free-carrier recombination. A qualitative observation of the temperature-dependent spectra clearly shows a transition at about $70 \mathrm{~K}$ between two completely different types of lineshapes, thus supporting the idea that two different PL mechanisms dominate the low-temperature and the hightemperature conditions. The blueshift with increasing excitation is attributed to a gradual filling of the energy states within the band tails. A broad emission band separates from the main emission peak at about $50 \mathrm{~K}$, redshifts with increasing $T$ and gradually disappears. This lower energy emission can 
Effects of thermal annealing on the optical properties of InGaNAs/GaAs multiple quantum wells

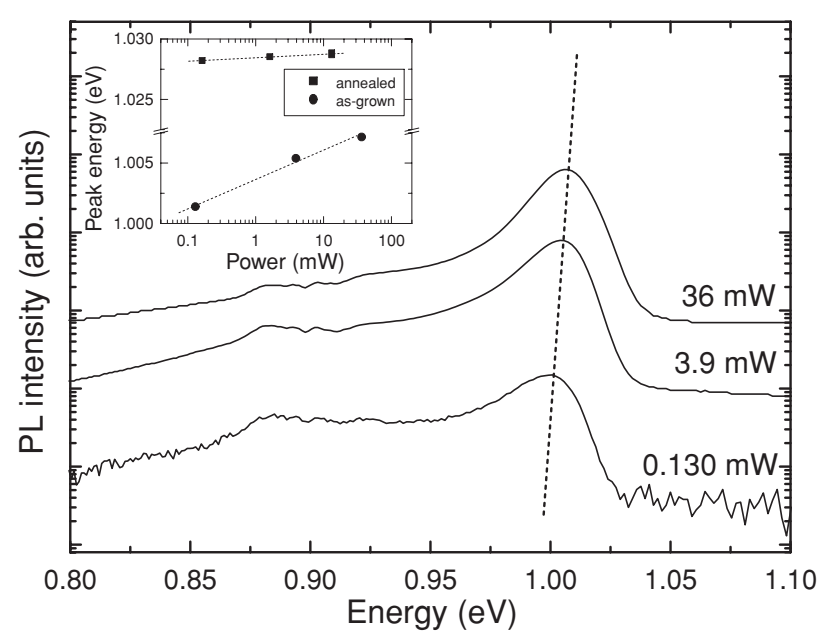

Figure 3. PL spectra of the as-grown sample recorded at $T=10 \mathrm{~K}$ under different excitation powers; the peak energy blueshift is indicated by the dashed line. The inset shows the peak energy of the PL spectra $(T=10 \mathrm{~K})$ versus the excitation power, for the as-grown and the annealed samples.

be attributed to the recombination of carriers localized at defect states and, as it is more evident from the inspection of the annealed sample spectra (which can be described at low temperatures by the convolution of two Gaussian bands), it is about $20 \mathrm{meV}$ under the fundamental gap emission. Several observations are reported in the literature about extrinsic emissions for InGaAs epitaxial layers or InGaAs/GaAs QWs with an indium concentration comparable to that of our sample; extrinsic transitions in the range 13-25 meV under the InGaAs bandgap have been found to involve $\mathrm{C}$ or $\mathrm{Zn}$ as acceptors [11]. A similar extrinsic mechanism is likely to produce the impurity-related emission observed in our samples.

The $10 \mathrm{~K}$ spectrum of the annealed sample (figure $2(b)$ ) shows some important differences compared to the as-grown one: besides the above-discussed blueshift of the emission peak: (i) the PL intensity is four times more intense; (ii) the FWHM is reduced (it is $13 \mathrm{meV}$ on the average); (iii) the lowenergy tail has disappeared. A much smaller blueshift of the emission peak is observed with increasing excitation power (inset of figure 3). The temperature-dependent spectra show a blueshift of the emission peak until about $50 \mathrm{~K}$, followed by a redshift with increasing $T$; a change in the lineshape of the PL peak occurs at about $80 \mathrm{~K}$. The lower energy side of the PL peak, probably related to impurity states, comes out gradually with increasing $T$ and it appears as less broad and less intense than in the as-grown sample. These results indicate that in the annealed sample the enhancement of the PL intensity and the reduction of the linewidth can be attributed to a removal of defects and in particular of $\mathrm{N}$-induced nonradiative centres from the InGaNAs layers. The disappearance of the lowenergy tail suggests that the annealing eliminates the deeper defects or alloy potential fluctuations. The smaller blueshift confirms that there is a less extended tail of localized states, related to a lower concentration of defects or to reduced compositional fluctuations of the alloy.

In order to get a further insight into the PL mechanisms of the two samples and the modifications induced by the annealing, we performed a systematic analysis of

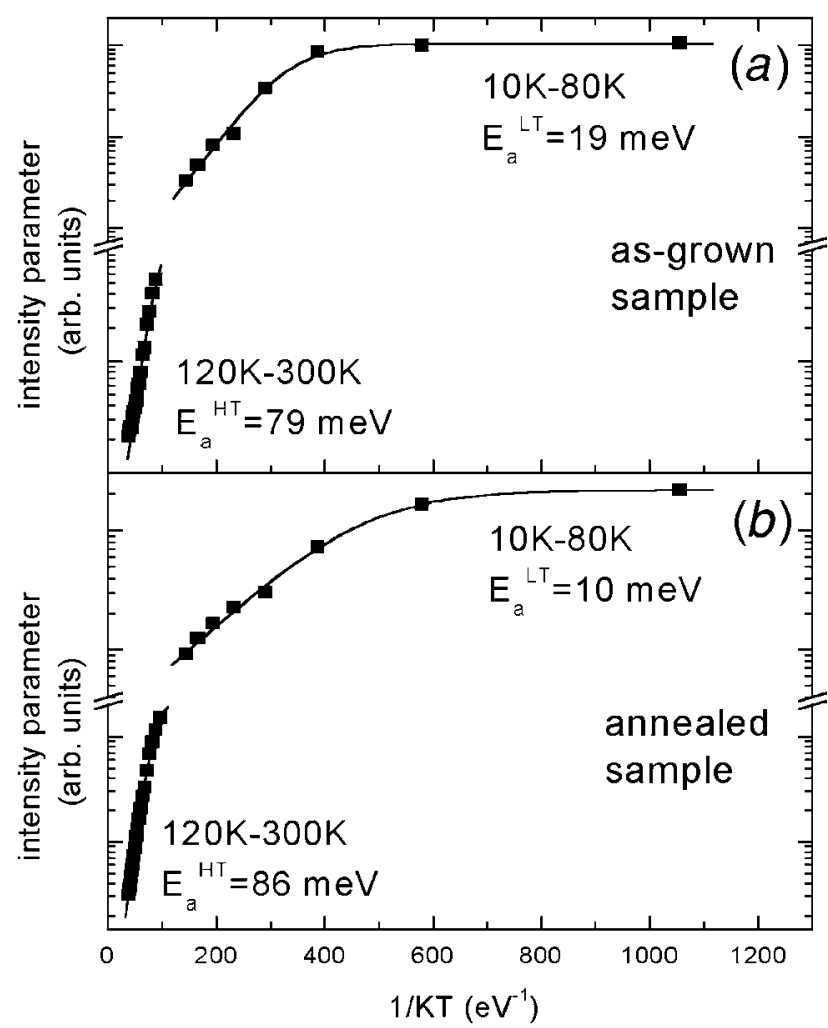

Figure 4. (a) Arrhenius plots of the integrated PL intensity (in the range $10-80 \mathrm{~K}$ ) and of the intensity parameter $I_{\mathrm{c}}$ (in the range 120-300 K), for point A of the as-grown sample. (b) The same for point $C$ of the annealed sample. The error bars for the experimental data are not visible in the figure scale.

the temperature-dependent spectra. In both samples, we distinguished two temperature ranges, characterized by two different thermal activation behaviours:

(i) The low-temperature range $(10-80 \mathrm{~K})$, in which the predominant PL mechanism is the localized carrier recombination. In all the four points of the as-grown sample, using Gaussian fits of the PL emission we obtained an Arrhenius plot of the integrated PL intensities providing the activation energy $E_{a}^{L T}$ for the PL thermal quenching. The experimental data and their fit for point $\mathrm{A}$ of the as-grown sample are shown in figure 4(a). We find that $E_{a}^{L T}$ decreases from $20 \mathrm{meV}$ at the centre of the wafer to about $10 \mathrm{meV}$ at the edge. A comprehensive list of our results is presented in table 1. We also fit the temperature dependence of the energy gap to a Varshni law (figure 5(a)). The comparison between the Varshni curve and the experimental data shows the presence of carrier localization at low temperatures and provides the localization energy $E_{l o c}$ (table 1). This is found to be very close to $E_{a}^{L T}$, suggesting that the PL peak quenching in the low-temperature range is dominated by an activation energy whose value is, in each point, comparable to the corresponding localization energy. A similar analysis of the annealed sample spectra in the same range of temperatures (figures $4(b)$ and $5(b)$ ) has given the results quoted in table 2 . The smaller average carrier localization indicates that the 
Table 1. The activation energy $E_{a}^{L T}$ for the PL thermal quenching in the low-temperature range (10-80 K), the localization energy as determined from the Varshni fit of the QW ground transition energy versus $T$, and the activation energy $E_{a}^{H T}$ for the PL thermal quenching in the high-temperature range $(120 \mathrm{~K}-300 \mathrm{~K})$, for the four investigated points of the as-grown sample.

\begin{tabular}{llll}
\hline & $E_{a}^{L T}(\mathrm{meV})$ & $E_{l o c}(\mathrm{meV})$ & $E_{a}^{H T}(\mathrm{meV})$ \\
\hline Point A & 19 & 23 & 79 \\
Point B & 20 & 21 & 83 \\
Point C & 11 & 16 & 85 \\
Point D & 14 & 17 & 73 \\
\hline
\end{tabular}

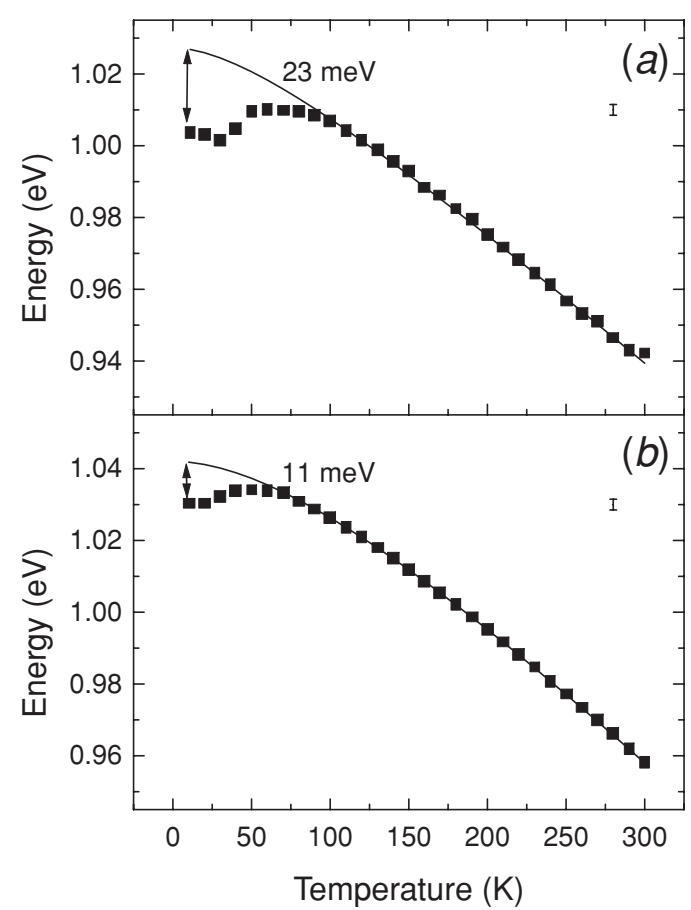

Figure 5. Temperature dependence of the QW fundamental transition energy for the point A of the as-grown sample $(a)$ and for the point $\mathrm{C}$ of the annealed sample $(b)$. The error bar is reported. The solid lines represent the Varshni curves which best fit the high-temperature data.

annealing induces a reduction of the average depth of the potential fluctuations. Hence, even though important potential fluctuations persist after the annealing, a reduction of the inhomogeneity at a microscopic scale has been induced. More importantly, the localization energy becomes more uniform across the wafer (about $10 \mathrm{meV}$ ), indicating that the annealing improves the uniformity of the sample on the long range $(\mathrm{cm})$.

(ii) The high-temperature range (120-300 K), dominated by the free-carrier recombination. For both samples, with increasing temperature the lineshape gradually loses the Gaussian profile. A high-energy tail appears, which is due to a thermal band filling effect. After a transition range, from about $120 \mathrm{~K}$ on, the lineshape can clearly be modelled by an analytical expression taking into account the contribution from the continuum states, expressed by a broadened step-like two-dimensional density of states
Table 2. The same as table 1 for the annealed sample.

\begin{tabular}{lrll}
\hline & $E_{a}^{L T}(\mathrm{meV})$ & $E_{l o c}(\mathrm{meV})$ & $E_{a}^{H T}(\mathrm{meV})$ \\
\hline Point A & 9 & 10 & 74 \\
Point B & 7 & 12 & 76 \\
Point C & 10 & 11 & 86 \\
Point D & 7 & 9 & 75 \\
& & & \\
\hline
\end{tabular}

and a two-dimensional Sommerfeld factor [12]:

$$
\begin{aligned}
I(\hbar \omega) & =I_{c} \omega^{2} \exp \left[-\left(\hbar \omega-E_{c}\right) / k T\right] \\
& \times \frac{1}{1+\exp \left[-\left(\hbar \omega-E_{c}\right) / \Gamma_{c}\right]} \\
& \times \frac{2}{1+\exp \left[-2 \pi \sqrt{E_{b} /\left|\hbar \omega-E_{c}\right|}\right]}
\end{aligned}
$$

where $E_{c}, \Gamma_{c}$ and $I_{c}$ are the energy of the intersubband transition, the related broadening parameter and the intensity parameter, respectively, and are assumed as free parameters in the calculations. $E_{b}$ is the exciton binding energy. We have assumed $E_{b}=7 \mathrm{meV}$, which is the value of exciton binding energy in InGaAs/GaAs QWs with similar well width and indium content [13].

We have then investigated the temperature dependence of the intensity parameter of the equation (1) that we have used to fit the PL spectra in the high-temperature range. We have extracted a new activation energy $E_{a}^{H T}$ for the PL thermal quenching from the Arrhenius plots of this intensity parameter (figures $4(a)$ and $(b)$ ). Our results, quoted in tables 1 and 2, clearly show that $E_{a}^{H T}$ is quite uniform across the sample, and it does not change in the two samples (about $80 \mathrm{meV}$ ). Such a value corresponds to the calculated energy barrier experienced by the ground level hole in the QW (about $90 \mathrm{meV}$ ). A much larger value of about $370 \mathrm{meV}$ is obtained for the confined electrons (these values agree well with recent experimental data [14]). Thus, in the high-temperature range, the PL quenching is due to the thermal escape of the holes from the QW. Both the well-width thinning and the proposed alloy composition variations would not be in contradiction with the observed uniformity of the $E_{a}^{H T}$ parameter along the two samples, because this activation energy is related to the detrapping of the holes, whose eigenvalues are much less influenced by variations of layer thickness and changes of $\mathrm{N}$ or In concentration than the electronic states.

\section{Conclusions}

In conclusion, we have performed a spatially resolved optical characterization of as-grown and thermally annealed InGaNAs/GaAs MQW samples, in order to clarify the effects of the thermal annealing on the optical properties of the sample and on their spatial homogeneity. We found that the annealing produces a blueshift of the PL peak of about $15 \mathrm{meV}$, and a considerable improvement of the optical properties of the sample: namely the enhancement of the PL intensity, a reduction of the linewidth and the disappearing of the lowenergy tail. These are related to some structural changes induced by the annealing, such as the reduction of the density 
of defects and of the average depth of the alloy potential fluctuations, and the improved homogeneity of the sample on the long range.

\section{Acknowledgments}

This paper was in part funded by the EU under BriteEuram BRPR-CT98-0721 ('OPTIVAN') and IST-199910450 ('GSQ'). AYuE gratefully acknowledges support by the A V Humboldt foundation.

\section{References}

[1] Weyers M, Sato M and Ando H 1992 Japan. J. Appl. Phys. 31 L853

[2] Xin H P and Tu C W 2000 Appl. Phys. Lett. 772870

[3] Shan W, Walukiewicz W, Ager III J W, Haller E E, Geisz J F, Friedman D J, Olson J M and Kurtz S R 1999 Phys. Rev. Lett. 861221

[4] Wei S H and Zunger A 1996 Phys. Rev. Lett. 76664
[5] Polimeni A, Capizzi M, Geddo M, Fischer M, Reinhardt M and Forchel A 2000 Appl. Phys. Lett. 772870

[6] Grenouillet L, Bru-Chevalier C, Guillot G, Gilet P, Duvaut P, Vannuffel C, Million A and Chenevas-Paule A $2000 \mathrm{Appl}$. Phys. Lett. 762241

[7] Xin H P, Kavanagh K L, Kondow M and Tu C W 1999 J. Cryst. Growth 201-202 419

[8] Pan Z, Li L H, Zhang W, Lin Y W, Wu R H and Ge W 2000 Appl. Phys. Lett. 771280

[9] Kitatani T, Nakahara K, Kondow M, Uomi K and Tanaka T 2000 J. Cryst. Growth 209345

[10] Riechert H, Yu Egorov A, Lifshits D, Borchert B and Illek S 2000 Nanotechnology 11201 and references therein

[11] See e.g. Fry K L, Kuo C P, Cohen M and Stringfellow G B 1985 Appl. Phys. Lett. 46955

Devine R L S and Moore W T 1988 Solid State Commun. 6519

[12] Cingolani R and Ploog K 1991 Adv. Phys. 40535

[13] Lee K-S, Aoyagi Y and Sugano T 1992 Phys. Rev. B 10269 Amand T H, Marie X, Barrau J, Brousseau M, Dunstan D J, Emery J Y and Goldstein L 1992 J. Appl. Phys. 722077

[14] Hetterich M, Dawson M D, Yu Egorov A, Bernklau D and Riechert H 2000 Appl. Phys. Lett. 761030 